\title{
Spinal Segmental Myoclonus Masquerading as a Psychogenic Movement Disorder
}

\author{
Laxmi Khanna ${ }^{1 *}$, Anuradha Batra ${ }^{1}$ and Ankita Sharma ${ }^{2}$ \\ ${ }^{1}$ Department of Neurology and Neurophysiology, Sir Gangaram Hospital, New Delhi, India \\ ${ }^{2}$ Department of Neurophysiology, Sir Gangaram Hospital, New Delhi, India
}

*Corresponding author: Dr. Laxmi Khanna, Consultant Neurologist and Neurophysiologist, Department of Neurology and Neurophysiology, Sir Gangaram Hospital, New Delhi-110060, India, Tel: 9873558121; E-mail: laxmikhanna@hotmail.com

Rec Date: December 26, 2018; Acc Date: January 05, 2019; Pub Date: January 11, 2019

Citation: Khanna L, Batra A, Sharma A (2019) Spinal Segmental Myoclonus Masquerading as a Psychogenic Movement Disorder. J Neurol Neurosci Vol.10 No.1:282.

\section{Abstract}

Spinal generated movement disorders are uncommon. An elderly gentleman presented with distressing jerks of both lower limbs which caused him much social embarrassment. He had received psychiatric treatment for these abnormal muscular spasms without relief. He had become depressed and withdrawn when he first presented to our outpatient department. A routine clinical examination followed by a long-term video EEG with simultaneous EMG clinched the diagnosis of a spinal segmental myoclonus. He underwent spinal decompression surgery with fixation followed by treatment with levetericetam and clonazepam which relieved his symptoms. Spinal generated movement disorders are uncommon and are often attributed to a psychiatric disorder in the absence of a better explanation. We take this opportunity to elaborate on the case details, investigations and the treatment of spinal myoclonus.

Keywords: Spinal myoclonus; Psychogenic movement; Depression

\section{Introduction}

Spinal segmental myoclonus is a rare movement disorder characterized by sudden involuntary contraction of groups of agonist and antagonist muscles supplied by contiguous segments of the spinal cord. An awareness of this clinical condition is essential as it is often mistaken to be a psychogenic condition. An elderly man was referred to our department with a probable psychogenic movement disorder in whom a diagnosis of spinal segmental myoclonus was made. We briefly mention the clinical features, pathophysiology and the treatment of spinal segmental myoclonus.

A 63-year-old, non-smoker, complained of distressing involuntary movements of both legs causing him to withdraw from society and become depressed. His symptoms started insidiously, three years ago with an uncomfortable sensation in his lower back followed by a painless involuntary rhythmic forward jerking of his legs which lasted for thirty seconds (Figures 1 and 2). These attacks occurred at rest and before falling asleep. The jerks increased in frequency and intensity curtailing his social life. There was no past history of any trauma, myelitis, demyelination or infections with human immunodeficiency virus.

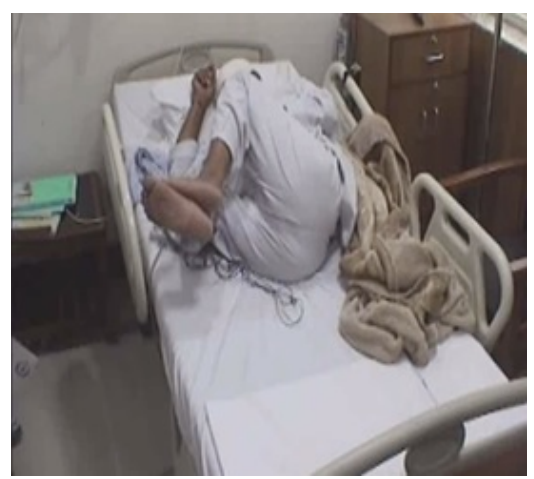

Figure 1 Sudden axial flexion jerk involving the hips, thighs and legs (Spinal segmental myoclonus).

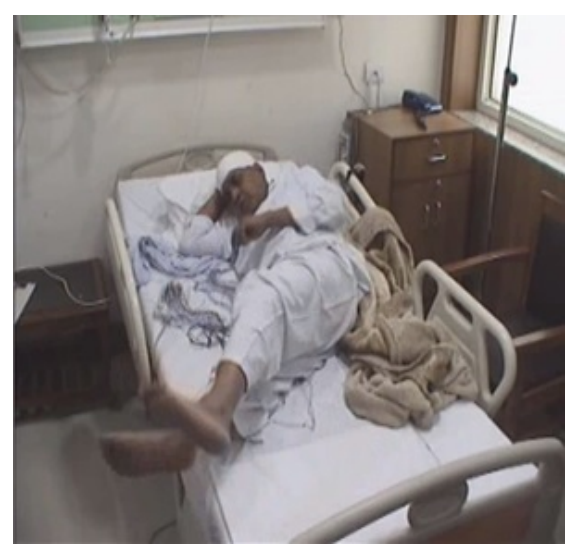

Figure 2 Dorsiflexion of both ankles (Spinal segmental myoclonus). 
On examination, he was well built and nourished with normal vital signs. His higher mental functions and cranial nerve examinations were normal. His muscle bulk and tone were normal in all four limbs and there were no fasciculations. Muscle power was $5 / 5$ in all four limbs proximally, in both upper limbs and right lower limb distally. The power of left knee extensors and foot dorsiflexors was 4/5. There was reduced touch and pain sensation over left L5-S1 distribution, and the left plantar reflex was not elicitable. Other superficial and other deep tendon reflexes were normal. There was no startle myoclonus or myoclonic jerks during sleep.

During his hospital stay, this gentleman experienced several involuntary rhythmic contractions of both legs. The movements were characterized by a sudden brief flexion of the trunk and hips with the thighs and legs bending forwards and ankles dorsiflexing (Figures 1 and 2). The patient was fully conscious, alert and awake during the attack. These jerks occurred in the recumbent position, but he could lose his balance if they occurred on standing. These attacks were not stimulus sensitive and could be provoked by stress.

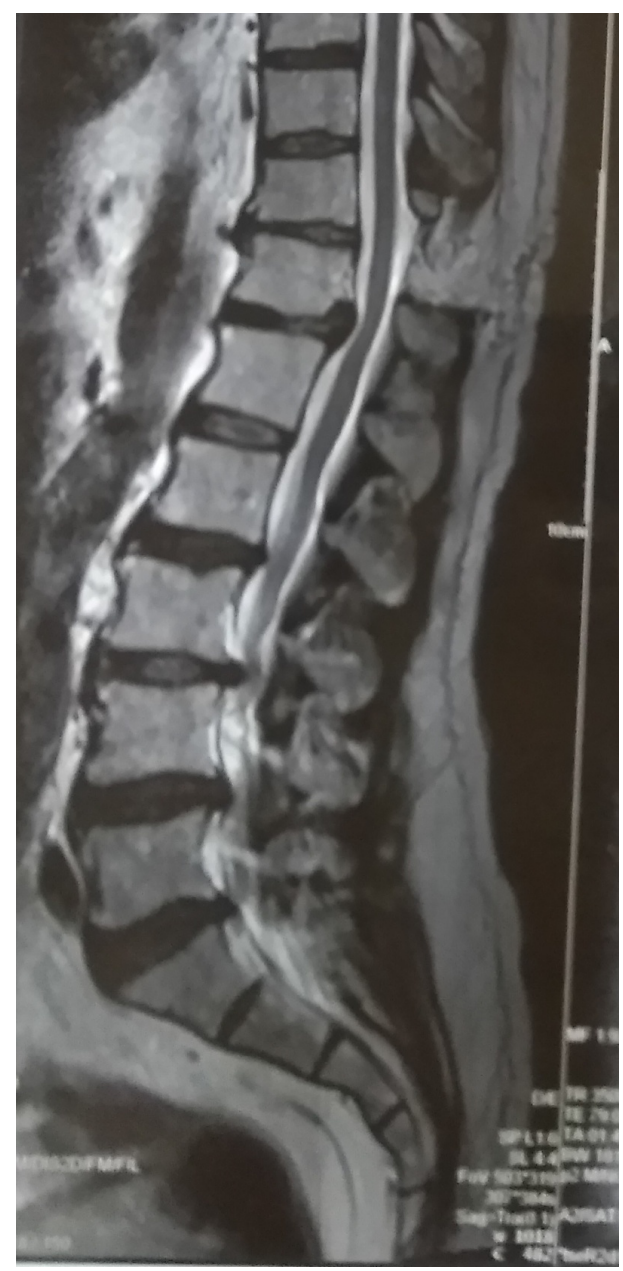

Figure $3 \mathrm{MRI}$ of dorsolumbar spine: A broad-based posterior and foraminal disc bulge at D12 - L1 level with ligamentum flavum thickening causing thecal sac indentation and compression of bilateral exiting roots.(white arrow) Multi level posterior disc at L4 - 5, L5 - S1.
His routine hemogram and biochemistry were normal. His nerve conduction study revealed an asymmetrical sensory motor axonal polyneuropathy involving both lower limbs. Routine EMG studies showed chronic reinnervation in proximal and distal muscles of both legs in a L4-S1 distribution. He underwent long term video EEG with simultaneous EMG recording from the left Tibialis anterior muscle. Myoclonic jerks of both lower limbs were recorded with no EEG correlate. During jerking, EMG recording showed persistent contractions of the left tibialis muscle occurring at frequency of 4-8 bursts per second, with each burst comprising of 4-5 motor unit discharges. The burst of tibialis anterior muscle activity was involuntary and was absent during voluntary leg movements. MRI of the dorsolumbar spine showed a broad-based posterior disc bulge at D12-L1 level with ligamentum flavum thickening causing thecal sac indentation and compression of bilateral exiting roots with a canal diameter of $1.0 \mathrm{~cm}$. There were multi-level small posterior disc bulges at L2 - L3, L3-4, L4-5 and L5-S1 without compression of the exiting nerve roots (Figure 3). This patient underwent spinal decompression surgery with fixation, and he was treated with clonazepam and levetericetam for the myoclonic jerks. Postoperatively, had a significant relief of his symptoms. He had monthly visits to the outpatient clinic. The rhythmic jerks had disappeared in a month and he was cheerful and optimistic. He was continued on levetericetam $500 \mathrm{mg}$ twice daily with clonazepam $0.5 \mathrm{mg}$ at bedtime with a plan to wean off after six months.

\section{Discussion}

Myoclonus has a prevalence of 8.6 cases per 100,000 per year [1]. Friedreich in 1881 first described myoclonus of spinal origin and since then there have been several similar case reports in the literature [1]. Involuntary rhythmic movements of the limbs have been noted in patients with spinal diseases caused by trauma, tumour, demyelination, arteriovenous malformations, degenerative spinal diseases, viral infections, intra thecal medication, antibiotics and regional anaesthetics $[1,2]$. There are rare case reports of spinal myoclonus in vitamin B 12 deficiency, post spinal radiation and in amyotrophic lateral sclerosis [1,2]. This condition may often be misdiagnosed as a psychogenic movement disorder if one is not aware of this abnormal phenomenon [1].

Spinal segmental myoclonus is characterized by brief, myoclonic jerks in a few spinal segmental myotomes with or without a rhythmic component $[3,4]$. The myoclonic movements are not elicited by external stimuli and occur in the awake state $[3,4]$. These movements affect the lower limbs more than the upper limbs [3]. Howell et al. attributed the pathophysiology of spinal myoclonus to a loss of spinal inhibitory interneuronal function that led to spontaneous repetitive discharges from the anterior horn cells [5]. This resulted in an exaggerated flexion reflex caused by decreased spinal inhibition, increased anterior horn cell excitability, aberrant axonal reexcitation and loss of inhibition from suprasegmental descending pathways [3-5]. Demyelinated axons caused axono-axonal ephaptic transmission within the damaged spinal cord [3-5]. These spontaneous discharges led 
to a co-contraction of the segmental agonist and antagonist muscles contributing to a spinal segmental myoclonus $[1,3,4]$. Calanchie et al. demonstrated bilateral simultaneous contractions in the flexors and extensors of the legs caused by reciprocal activation of agonist and antagonist muscles which evolved in to a rhythmic spinal generated movement [3]. The burst of EMG activity in the left tibialis anterior represented an example of this type of involuntary muscular contraction.

\section{Conclusion}

In spinal segmental myoclonus, there is a deficiency of inhibitory glycinergic transmission which leads to altered spinal circuits and the development of a myoclonic focus within the spinal cord [6]. Levetiracetam reverses the inhibition of glycine and GABA gated currents which increase the glycinergic transmission within the spinal cord and dampens the myoclonic movements [6]. This patient, underwent a laminectomy at D11-12 with spinal fixation. He was treated with a combination of Clonazepam and Levetricetam which abolished the myoclonic jerks. This rare movement disorder was initially considered psychogenic but a long-term video EEG with simultaneous EMG recording proved that the movement was due to a spinal segmental myoclonus and the patient responded well to the treatment.

\section{References}

1. Ray BK, Guha G, Misra AK, Das SK (2005) Involuntary jerking of lower half of the body (Spinal myoclonus). JAPI 53: 141-143.

2. Hopkins AP, Micheal WF (1974) Spinal myoclonus. J Neurol Neurosurg Psychiatry 37: 1112-1115.

3. Calanchie B (2006) Clinical notes: Spinal myoclonus after spinal cord injury. J Spinal Cord Med 29: 413-424.

4. Termsarasab P, Thammongkolchai T, Frucht SJ (2015) Spinalgenerated movement disorders: A clinical review. Journal of Clinical Movement Disorders 2: 18-31.

5. Campos CR, Papaterra JC (2003) A case of primary spinal myoclonus. Arq Neuropsiquiatr 61: 112-114.

6. Keswani SC, Kossoff EH, Krauss GL, Hagerty C (2002) Amelioration of spinal myoclonus with levetericetam. J Neurol Neurosurg Psychiatry 73: 457. 